\title{
Development of synaptic connectivity in the retinal direction selective circuit.
}

Ryan D. Morrie ${ }^{1}$ and Marla B. Feller ${ }^{1,2 *}$

${ }^{1}$ Department of Molecular and Cell Biology, University of California, Berkeley, Berkeley, California 94720-3200

${ }^{2}$ Helen Wills Neurosciences Institute, University of California, Berkeley, Berkeley, California 94720-3200

${ }^{*}$ Correspondence should be addressed to:

Marla B. Feller, Ph.D.; Department of Molecular and Cell Biology \& Helen Wills

Neuroscience Institute; University of California, Berkeley, 142 Life Sciences Addition - 3200, Berkeley, CA 94720-3200

$\underline{\text { mfeller@berkeley.edu }}$ 


\begin{abstract}
Direction selectivity is a classic neuronal computation that has been described in many different sensory systems. The circuit basis of this computation is perhaps best understood in the retina, where direction selectivity is the result of asymmetric connectivity patterns between excitatory and inhibitory circuit components. Retinal direction selective circuits emerge prior to eye-opening, though components of the circuit undergo refinement after vision begins. These features make the direction selective circuit a rich model in which to investigate neuronal circuit assembly. In this Opinion, we highlight recent experiments investigating the contribution of various molecular cues, as well as neuronal activity, to the development of the retinal direction selective circuit.
\end{abstract}

\title{
Introduction: organization of the retinal direction selective circuit
}

A classic goal in developmental neurobiology is to understand how molecular markers and neuronal activity interact to instruct the emergence of functional circuits (reviewed in [1]). To tease apart these contributions, it is vital that the detailed wiring diagram of the circuit and its computation are well understood. To this end, the retinal direction selective circuit is an excellent system to examine this developmental puzzle.

There are two key features of direction selective circuits that emerge during development. First is their tuning. Direction selective ganglion cells (DSGCs) fire robustly to stimuli moving in a preferred direction and weakly to stimuli moving in the opposite, or null direction. (Though there are several types of DSGCs, in this review we focus on one class, On-Off DSGCs, whose members respond to both increments and decrements in luminance). Second, the preferred directions of DSGCs are not randomly distributed, but rather cluster tightly along particular directions of motion [2,3]. This results in DSGCs subpopulations with preferred directions along four axes: nasal, temporal, dorsal, and ventral, which we refer to as the cardinal directions (Figure 1B,D).

The circuit components that underlie DSGC tuning are well identified at the cellular level: a class of inhibitory interneurons, called starburst amacrine cells (SACs), provide GABAergic inhibition, while a class of excitatory interneurons, called bipolar cells, provide glutamatergic excitation (Figure 1A). Directional tuning arises predominantly via asymmetric inhibitory signaling; when inhibition from SACs is abolished using GABA-A antagonists [4], ablation of SACs [5], or reversible pharmacogenetic silencing [6] DSGCs fire to stimuli moving in their null direction, eliminating direction selectivity. A role for excitation in generating direction selectivity is more controversial. Calcium imaging in bipolar cell terminals $[7,8]$ and imaging of glutamate released from bipolar cells [9] in response to visual stimulation indicate that bipolar cells do not encode direction. However, a recent studied implicated a role for excitation in direction selectivity. In a transgenic mouse with significantly reduced GABA release from SACs, a substantial number of DSGCs remained [10]; the DSGCs that retained direction selectivity did so via an asymmetric excitatory input [10], demonstrating a role for excitation in this computation in at least a subset of DSGCs. Indeed, transgenic manipulations of cone bipolar cell $(\mathrm{cBC})$ release and connectivity can have large effects on the direction selective circuit [11,12].

Importantly, the bipolar cell subtypes, SACs, and DSGCs that comprise On-Off direction selective circuits are all symmetric in their morphology (see Figure 1D for 
exception) and therefore direction selectivity emerges as the result of selective wiring. Several experimental approaches have converged onto a consistent description of the inhibitory wiring scheme that underlies direction selectivity. Paired SAC-DSGC recordings revealed that SACs located on the null side of a DSGC have a stronger GABAergic synaptic conductance than SACs located on the preferred side [13-15]. Serial electron microscopy reconstructions revealed the subcellular connectivity pattern underlying this asymmetry; the orientations of individual SAC processes strongly correlated with the null direction of the DSGC with which they formed synapses. Thus, null-oriented SAC processes form more synapses with DSGCs than preferred-oriented SAC processes [16] (Figure 1C). Moreover, the clustering of DSGC preferred directions along cardinal axes functions as a constraint on this antiparallel wiring of SACs onto DSGCs (Figure 1D). The asymmetric inhibitory input from SACs is further enhanced by the intrinsic direction selectivity of the SAC process itself to centrifugal motion [7,1719]. Antiparallel wiring (Figure 1D) dictates that null direction motion provides centrifugal stimulation for null oriented SAC processes. Hence, these subcellular mechanisms work together to ensure object motion in the null direction induces a larger release of GABA from SACs onto DSGCs than motion in the preferred direction.

Although the basis of excitation-driven direction selectivity is not well understood, a variety of approaches, including EM reconstructions [20], viral tracing [7], and electrophysiological recordings [8], have revealed asymmetries in bipolar cell inputs between SACs and DSGCs, despite their similar stratification patterns. For example, cone bipolar cell type 7 (cBC7) contacts ON SACs but not DSGCs. In addition, the centrifugal direction selectivity of SACs themselves may be partially determined by specific subcellular connectivity patterns with cBCs [21-23].

Despite recent advances in our detailed understanding of the mature DS circuit $[3,24]$, an essential developmental question remains: what instructs the selective synaptic connectivity that underlies the antiparallel wiring of SACs onto DSGCs? Here we describe recent progress in elucidating when direction selective circuit elements emerge, the molecular cues that are involved in their development, and evidence that neuronal activity may play an important role in direction selective circuit maturation.

\section{Timeline of DS circuit development}

DS circuits emerge early in retinal development. Using MEA and cell attached recordings, direction selective responses have been observed at eye opening (end of the second postnatal week in mice), and even a few days prior [14,25-28]. At this time bipolar cells are beginning to link photoreceptors to the rest of the retina, indicating that the antiparallel wiring of SACs onto DSGCs exists prior to the maturation of bipolar cell synapses.

When does asymmetric SAC-DSGC wiring emerge? Transgenic mouse lines that label specific subpopulations of DSGCs (reviewed in [29], Figure 1D) enable identification of DSGCs with particular preferred directions prior to the establishment of functional light responses in the retina. Paired whole cell recordings between transgenically labeled DSGCs and SACs revealed that asymmetries in inhibition begin near the end of the second postnatal week [14,30] and channelrhodopsin activation of SACs showed asymmetric connectivity with DSGCs as early as P9 [31]. By isolating quantal GABA release events from SAC-DSGC pairs, we found that this asymmetric 
conductance emerges due to an increase in the number of synapses from null-oriented SAC processes, rather than a change in synaptic strength [30]. Hence, the early development of the DS circuit involves a process of rapid and selective synaptogenesis between SACs and DSGCs prior to light response maturation.

\section{Towards a molecular understanding of direction selectivity}

Specific genetic fingerprints are postulated to exist for each of the different DSGC subpopulations that have been identified in transgenic mice [32]. Indeed, microarray expression profiling identified candidate genes hypothesized to be specifically expressed in ventral motion preferring DSGCs (BD) [33] or SACs [34]. After analyzing the expression of these candidates in two mouse lines that labeled nasal motion preferring DSGCs (Drd4-GFP, W9-YFP) [34,35], Kay et al., found that among DSGCs, MMP17 was expressed exclusively by nasal preferring cells, while Cdh6 and Col25a1 were expressed exclusively by ventral and dorsal preferring DSGCs. These molecular signatures were present a week before eye opening [34] and in some cases immediately following differentiation [36], further indicating that DSGCs differentiate into cardinal direction subpopulations with specific genetic fingerprints long before the maturation of light responses.

The presence of specific genetic markers for DSGC subpopulations leads to the hypothesis that each DSGC subpopulation has a unique molecular signature that is recognized by antiparallel SAC processes expressing the requisite complementary molecular cues (Figure 1D). Here we describe multiple molecules that are essential for the proper development of components of the direction selective circuit.

\section{Molecularly induced alterations in SAC morphology}

In looking for SAC-DSGC synaptogenesis factors, members of two classic synaptogenesis gene families were found to be highly expressed in SACs, the protocadherin- $\gamma$ cluster [37] and the semaphorin Sema6A [38]. Both of these genes are essential for proper SAC morphological development and function.

Protocadherins are responsible for dendritic self-avoidance in SACs and cerebellar Purkinje cells [37]. The protocadherin- $\gamma$ cluster has a unique genetic structure whereby different extracellular variants of protocadherin- $\gamma$ proteins are expressed stochastically in individual cells. The model is that homotypic interactions between protocadherin isoforms cause self-avoidance but heterotypic interactions do not [39]. Protocadherin- $\gamma$ cluster deletion in SACs $\left(P c d h g^{--}\right)$resulted in a collapse of SAC 'starburst' morphology and increased SAC-SAC connectivity (Figure 2B), presumably via an absence of self-avoidance. In contrast, directed SAC expression of a single protocadherin- $\gamma$ isoform $\left(P c d h g^{3}\right)$ in the $P c d h g^{-/}$background resulted in a restoration of self avoidance, but an absence of SAC-SAC synapses because of increased non-self avoidance (Figure 2C) [37,40]. Thus, Pcdhg heterogeneity amongst SACs is required for normal development of SAC-SAC connectivity.

What is the impact of altered SAC-SAC connectivity on direction selectivity? Ventral motion preferring DSGCs (HB9-GFP) in the $P c d h g^{-/}$mouse have reduced directional tuning and a wider distribution of preferred directions (Figure 2B). In contrast, in $P c d h g^{3}$ mice the ventral motion preference was maintained, but the strength of direction selectivity was reduced (Figure 2C). The reduced direction selectivity in both 
cases resulted from weaker and more symmetric SAC-DSGC inhibition [40]. Hence, these data indicate that disrupting SAC-SAC connectivity results in reduced directional tuning.

Semaphorin mutants also link a change in SAC morphology to reduced direction selectivity. Semaphorins are a class of transmembrane proteins with multiple roles in synapse development and axon guidance, especially within the retina (reviewed in [41]). Sema6A is essential for the ON/OFF segregation found in the inner plexiform layer of the retina [42] and signals through PlexinA2 to achieve proper lamination of $\mathrm{ON}$ and $\mathrm{OFF}$ SAC dendrites [38]. In Sema6 $A^{-/}$mice, ON, but not OFF, SACs lose their morphological symmetry, and nasal preferring DSGCs (TrHr-GFP) exhibit reduced DS for bright, but not dark, moving edges [38] (Figure 3D).

These studies demonstrate that morphological alterations in SACs and/or SACSAC signaling results in compromised direction selectivity, likely via reduced direction selectivity in SACs themselves. Both mouse models exhibit degraded DS in genetically identified subpopulations of DSGCs due to increased null direction-evoked spiking activity. However, neither Pcdhg nor Sema6A has been implicated in patterning the asymmetric SAC-DSGC connectivity fundamental to the direction selective computation. Thus, it is predicted that loss of direction selective tuning in these mice is not specific to a particular subpopulation of DSGC.

\section{Molecular determinants of horizontal preferred directions}

A recent study identified for the first time a gene that selectively affects the development of some direction selective axes while others remain intact. Based on the observation that ablation of SACs abolishes the optokinetic reflex [5], Yonehara and colleagues studied mice with mutations in FRMD7, a gene linked to infantile idiopathic nystagmus, a disorder characterized by a loss of horizontal optokinetic reflexes in humans [43]. Mice with a hypomorphic mutation in FRMD7 (FRMD7 $\left.{ }^{\mathrm{tm}}\right)$ completely lack the optokinetic reflex and retinal DS responses along the horizontal axes, but retain normal responses along the vertical axes [44] (Figure 2E).

FRMD7 is expressed exclusively in SACs [44,45], but unlike in other mouse models with reduced direction selectivity [38,40], SACs in $F R M D 7^{t m}$ mice appeared morphologically and functionally normal [44]. Furthermore, temporal motion preferring DSGCs (Hoxd10-GFP) in FRMD ${ }^{t m}$ mice lost directional tuning due to a lack of spiking to temporally moving stimuli, instead of the increases in spiking to null direction stimuli

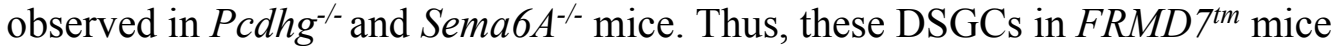
experience strong but symmetric GABAergic inhibition to horizontal motion stimulation [44].

The symmetric inhibition phenotype in $F R M D 7^{t m}$ mice leads to the hypothesis of a distinct developmental mechanism in which DSGCs may provide repulsive signals to preferred-oriented SAC dendrites, rather than attractive signals to null-oriented ones. FRMD7 itself is most likely not instructional for asymmetric wiring of SACs to DSGCs because it is expressed symmetrically in SAC dendrites [44]. However, it may be involved in the trafficking of other proteins to the correct subcellular location due to its interactions with actin cytoskeleton organizers [46].

\section{The role of activity in direction selective circuit development}


Thus far we have described roles for molecules involved in the wiring of inhibitory synapses that underlie direction selectivity. However, activity also influences the establishment, subcellular localization, and persistence of GABAergic synapses [47] in many parts of the brain. Hence, neuronal activity could affect three components of SAC-DSGC wiring in the DS circuit: 1) SAC-DSGC synaptogenesis, 2) symmetry breaking to produce anti-parallel SAC-DSGC wiring, or 3) refinement of SAC-DSGC wiring.

Strong SAC-DSGC inhibition emerges early in development, prior to the maturation of the light response [14,31], when the primary source of activity in the retina is retinal waves. Do retina waves play a role in SAC-DSGC synaptogenesis? Several studies based on pharmacological blockades $[14,28]$ or genetic alterations $[25,48]$ of retinal waves have not revealed an effect on direction selective responses at eye opening, indicating that SAC-DSGC synapses are not influenced by the patterned activity of retinal waves $[49,50]$. However, one can never be sure that the activity manipulations are functioning at all times (see Discussion in [48]) and therefore these negative results do not rule out a potential role for SAC-DSGC signaling in synaptogenesis.

The emergence of asymmetric SAC-DSGC synaptic connectivity also occurs prior to eye-opening. Therefore, the symmetry-breaking step is either "hard-wired" or influenced by spontaneous activity. In a recent study in hippocampus, it was shown that activity-dependent transcription factors played a role in the establishment of a subcellular GABAergic connectivity pattern onto pyramidal cells [51]. We speculate that a similar model may occur in the retina in which the presence of neuronal activity is required for the production of specific proteins in SACs that are then trafficked to specific SAC processes.

Importantly, the process of direction selective circuit development does not end at eye-opening. The strength of direction selective tuning is higher in retinas from adult mice than at eye opening [25,40,52,53,54] (but see [27]) and most strikingly, DSGC preferred directions do not cluster along the cardinal directions in young mice $[25,52,54]$ (Figure 3A). This clustering is a process of refinement; population calcium imaging of nasal preferring $\mathrm{GFP}^{+}$DSGCs has shown that their preferred directions are less clustered at eye opening compared to adults, but are never tuned to a direction more than $90^{\circ}$ from nasal (Figure 3A, inset) [54]. Furthermore, Bos et al., found that visual activity is required for this refinement process, as animals reared in the dark also exhibit a lack of DSGC preferred direction clustering (Figure 3A) [54]. Whether this reduced clustering in the absence of visual activity is a result of fewer postsynaptic GABAergic sites on bistratified RGCs [55], a failure to refine SAC-SAC connectivity (Figure 3C) [40], or imprecise SAC-DSGC connectivity remains to be determined.

\section{Conclusion:}

The retinal DS circuit provides a powerful model in which to study the patterning of synapses in neural circuits due to our strong understanding of the cell types and their connectivity patterns that comprise the circuit in the adult $[3,24]$. Thus far, only a small number of molecules that identify DSGC subpopulations, that are crucial for the normal development of SACs, or that are necessary for the development of direction selectivity of certain DSGC subpopulations have been identified. Thus, the question of what 
instructs the antiparallel wiring of individual SACs processes to DSGCs remains to be answered.

We end with a framework for determining how asymmetric SAC-DSGC wiring develops. In this hypothetical scenario, SACs sense one of the many molecular gradients that are found along cardinal axes in the developing retina (reviewed in [56,57]) and use them to traffic different proteins to their processes oriented in different directions. These proteins then interact with proteins expressed by the different classes of DSGCs in either a repulsive or attractive manner to prevent or induce synapse formation. Our hope is that novel candidates will be identified in unbiased screens, such as a recent one designed to look for cell surface recognition partners that identified a novel set of proteins governing SAC and DSGC dendritic lamination [58]. To probe effects on synaptic connectivity, candidate genes can be tested using cell specific manipulations to express or delete them presynaptically in bipolar cells and SACs (such as in[12,59]) or postsynaptically in subtypes of DSGCs (such as in [60]). Lastly, activity manipulations targeted to SACDSGCs signaling will be necessary to determine whether neuronal activity plays a role in the expression, trafficking or function of these candidate proteins. Only through such a multipronged approach will the puzzle of direction selective wiring be solved. 
Figure 1. Major components of the direction selective circuit.

A. Schematic of the identified cellular components in the direction selective circuit. Photoreceptors (top) synapse onto OFF bipolar cells (cone bipolar cell, cBC2 and cBC3) through sign-conserving synapses and onto ON bipolar cells (cBC5 and cBC7) through sign-inverting synapses. Bipolar cells provide excitatory gluatamatergic input to SACs and DSGCs: cBC2 provides about half the overall excitatory input onto DSGC OFF arbors while cBC5 provides the majority of excitatory input onto DSGC ON arbors [12]. Different bipolar cell subtypes contact different SACs at the subcellular locations shown $[21,22]$. Note the lack of bipolar cell contact on the distal portions of SAC processes [23]. SACs provide lateral GABAergic inhibition onto other SACs and feedforward GABAergic inhibition onto DSGCs. Note, SACs also provide cholinergic excitation to DSGCs. The role of this cholinergic synapse in the DS computation is an area of active research and is not covered in this review.

B. There are 4 subpopulations of On-Off DSCGs whose preferred directions cluster along the cardinal axes. Left Retina loaded with the calcium indicator OGB via electroporation. Circled cells demarcate ON-OFF DSGCs, with color representing the preferred direction cluster. Scale bar: $100 \mu \mathrm{m}$. Right Tuning curves and respective vector sums of $\mathrm{Ca}^{2+}$ signals in DSGCs in response to moving bar stimuli. $\mathrm{N}=$ nasal, $\mathrm{D}=$ dorsal, $\mathrm{T}=$ temporal, $\mathrm{V}=$ ventral in retinal coordinates. Figure adapted from [16].

C. SACs on the null side of DSGCs form more GABAergic synapses than SACs on the preferred side. Schematic depicting wiring diagram underlying directional GABA release onto DSGCs. The null-oriented processes of the null side SAC form many GABAergic synapses with the DSGC, while the preferred-oriented processes of the preferred side SAC rarely form GABAergic synapses with the DSGC.

D. Summary of proposed antiparallel SAC-DSGC wiring diagram. Each color represents specific molecular marker expressed by one of the four subpopulations of DSGCs, as well as the corresponding molecular markers expressed by antiparalleloriented SAC processes. The transgenic mouse lines listed express GFP or Cre in specific subpopulations of DSGCs. (Note we are not discussing ON DSGCs, which compute DS like ON-OFF DSGCS via GABA release from SACs, or OFF DSGCs, which compute DS through a mechanism independent of SACs [3,61-63]).

Figure 2. Summary of SAC morphology and connectivity defects in various mutant mouse lines and consequences for direction selectivity.

A. Left: SACs in WT mice have radially symmetric dendrites and form GABAergic synaptic connections onto other SACs. SAC connections onto DSGCs are not pictured. Right: Tuning curve vector sums with amplitude representing strength of tuning and the angle representing the preferred direction. For $\mathrm{Hb} 9-\mathrm{GFP}^{+}$DSGCs, which prefer ventral motion, both ON and OFF responses are strongly tuned. Figure adapted from [40]. B. SACs in mice lacking the entire $\gamma$-Protocadherin cluster do not exhibit self-avoidance, resulting in loss of radially symmetric morphology, and form GABAergic synapses with other SACs, as well as autapses. Both ON and OFF DS responses from $\mathrm{Hb} 9-\mathrm{GFP}^{+}$ DSGCs are weaker than in WT mice and are not consistently tuned toward the ventral direction. Figure adapted from [40]. 
C. SACs in mice expressing only one $\gamma$-Protocadherin isoform have radially symmetric morphology but display little dendritic overlap and rarely form GABAergic synapses with other SACs. Both ON and OFF DS responses from $\mathrm{Hb} 9-\mathrm{GFP}^{+}$DSGCs are weaker than in WT but generally tuned to the ventral direction. Figure adapted from [40]. D. ON SACs in mice lacking Sema6A do not have radially symmetric morphology, exhibit reduced self-avoidance, and have smaller overall dendritic arbors. They still receive GABAergic inhibition but SAC-SAC connectivity has not been tested. OFF SACs in this mouse have slightly smaller dendritic field areas but are otherwise normal (not shown). In Trhr-GFP ${ }^{+}$DSGCs, which prefer nasal motion, ON DS responses are weaker than in WT but generally tuned to the nasal direction, while OFF DS responses remain strongly tuned. Figure adapted from [38].

E. SACs in mice with a hypomorphic mutation in FRMD7 have normal morphology, but precise SAC-SAC connectivity has not been tested.

Middle: DS responses from MEA recordings of FRMD $7^{\text {tm }}$ retina explants show only ventrally and dorsally tuned DSGCs.

Right: Hoxd10-GFP ${ }^{+}$DSGCs, which prefer temporal motion in WT mice, do not fire action potentials in response to moving bars in $F R M D 7^{t m}$ mice. Figure adapted from [44].

Figure 3. Development of DS circuit components.

A. DSGC preferred directions do not cluster along cardinal directions in young and dark reared animals [52,54]. Tuning curve vector sums of calcium responses to moving bars for all DSGCs, with amplitude representing strength of tuning and the angle representing the preferred direction. Insets: Preferred directions of nasal preferring Drd4-GFP ${ }^{+}$DSGCs are less clustered along the nasal axis in young and dark reared animals. Figure adapted from [54].

B. SAC-SAC GABAergic synapses are refined during development. Although both SACs will form GABAergic synapses onto the other, for simplicity the red circles only indicate synapses from the black SAC onto the grey SAC.

Left: In young animals, SACs form GABAergic synapses onto other SACs regardless of inter-soma distance.

Right: In adult animals, SACs only form GABAergic synapses onto other SACs with an inter-soma distance $>100 \mu \mathrm{m}$. Schematic based on results from [40]. 


\section{Acknowledgements}

We thank Anna Vlasits for helpful comments on this manuscript. Support was provided by National Science Foundation (NSF) Grant DGE 1106400 (R.D.M.) and NIH ROI1EY019498 and RO1EY013528. 
1. Yogev S, Shen K: Cellular and molecular mechanisms of synaptic specificity. Annu. Rev. Cell Dev. Biol. 2014, 30:417-437.

2. Oyster CW, BARLOW HB: Direction-Selective Units in Rabbit Retina: Distribution of Preferred Directions. Science 1967, 155:841-842.

3. Vaney DI, Sivyer B, Taylor WR: Direction selectivity in the retina: symmetry and asymmetry in structure and function. Nat Rev Neurosci 2012, 13:194-208.

4. Wyatt HJ, Day NW: Specific effects of neurotransmitter antagonists on ganglion cells in rabbit retina. Science 1976, 191:204-205.

5. Yoshida K, Watanabe D, Ishikane H, Tachibana M, Pastan I, Nakanishi S: A key role of starburst amacrine cells in originating retinal directional selectivity and optokinetic eye movement. Neuron 2001, 30:771-780.

6. Vlasits AL, Bos R, Morrie RD, Fortuny C, Flannery JG, Feller MB, Rivlin-Etzion M: Visual stimulation switches the polarity of excitatory input to starburst amacrine cells. Neuron 2014, 83:1172-1184.

7. Yonehara K, Farrow K, Ghanem A, Hillier D, Balint K, Teixeira M, Jüttner J, Noda M, Neve RL, Conzelmann K-K, et al.: The first stage of cardinal direction selectivity is localized to the dendrites of retinal ganglion cells. Neuron 2013, 79:1078-1085.

8. Chen M, Lee S, Park SJH, Looger LL, Zhou ZJ: Receptive field properties of bipolar cell axon terminals in the direction-selective sublaminas of the mouse retina. J. Neurophysiol. 2014, 112:jn.00283.2014.

9. Park SJH, Kim IJ, Looger LL, Demb JB, Borghuis BG: Excitatory Synaptic Inputs to Mouse On-Off Direction-Selective Retinal Ganglion Cells Lack Direction Tuning. J. Neurosci. 2014, 34:3976-3981.

10. •Pei Z, Chen Q, Koren D, Giammarinaro B, Ledesma HA, Wei W: Conditional Knock-Out of Vesicular GABA Transporter Gene from Starburst Amacrine Cells Reveals the Contributions of Multiple Synaptic Mechanisms Underlying Direction Selectivity in the Retina. $J$. Neurosci. 2015, 35:13219-13232.

This study demonstrates a key role of excitatory signaling in the generation of DS. The authors found a substantial number of DSGCs remained in a transgenic mouse in which VGAT was specifically knocked out of SACs.

11. -Shi Z, Trenholm S, Zhu M, Buddingh S, Star EN, Awatramani GB, Chow RL: Vsx1 Regulates Terminal Differentiation of Type 7 ON Bipolar Cells. $J$. Neurosci. 2011, 31:13118-13127.

The authors localize expression of Vsx1 in ON bipolar cells to cBC7. They then show that the ON direction selectivity of DSGCs is decreased in $\mathrm{Vsx}^{-/-}$ 
mice due to increased null direction spiking, while OFF direction selective tuning remains normal.

12. ••Duan X, Krishnaswamy A, la Huerta De I, Sanes JR: Type II Cadherins Guide Assembly of a Direction-Selective Retinal Circuit. Cell 2014, 158:793-807.

This study uses optogenetic and molecular characterization of bipolar cell connectivity in the DS circuit to show that cadherins instruct the lamination of bipolar cells axons and their synaptic connections with SACs and DSGCs.

13. Fried SI, Münch TA, Werblin FS: Mechanisms and circuitry underlying directional selectivity in the retina. Nature 2002, 420:411-414.

14. Wei W, Hamby AM, Zhou K, Feller MB: Development of asymmetric inhibition underlying direction selectivity in the retina. Nature 2011, 469:402-406.

15. Lee $\mathrm{S}, \mathrm{Kim} \mathrm{K}$, Zhou ZJ: Role of ACh-GABA cotransmission in detecting image motion and motion direction. Neuron 2010, 68:1159-1172.

16. Briggman KL, Helmstaedter M, Denk W: Wiring specificity in the directionselectivity circuit of the retina. Nature 2011, 471:183-188.

17. Euler T, Detwiler PB, Denk W: Directionally selective calcium signals in dendrites of starburst amacrine cells. Nature 2002, 418:845-852.

18. Lee $\mathrm{S}$, Zhou ZJ: The synaptic mechanism of direction selectivity in distal processes of starburst amacrine cells. Neuron 2006, 51:787-799.

19. Hausselt SE, Euler T, Detwiler PB, Denk W: A dendrite-autonomous mechanism for direction selectivity in retinal starburst amacrine cells. Plos Biol 2007, 5:e185.

20. Helmstaedter M, Briggman KL, Turaga SC, Jain V, Seung HS, Denk W: Connectomic reconstruction of the inner plexiform layer in the mouse retina. Nature 2013, 500:168-174.

21. Kim JS, Greene MJ, Zlateski A, Lee K, Richardson M, Turaga SC, Purcaro M, Balkam M, Robinson A, Behabadi BF, et al.: Space-time wiring specificity supports direction selectivity in the retina. Nature 2014, doi:10.1038/nature13240.

22. Greene MJ, Kim JS, Seung HS, EyeWirers: Analogous Convergence of Sustained and Transient Inputs in Parallel On and Off Pathways for Retinal Motion Computation. CellReports 2016, doi:10.1016/j.celrep.2016.02.001.

23. Vlasits AL, Morrie RD, Tran-Van-Minh A, Bleckert A, Gainer CF, DiGregorio DA, Feller MB: A Role for Synaptic Input Distribution in a Dendritic Computation of Motion Direction in the Retina. Neuron 2016, 89:1317-1330. 
24. Helmstaedter M, Borst A: Common circuit design in fly and mammalian motion vision. Nat Neurosci 2015, doi:10.1038/nn.4050.

25. Elstrott J, Anishchenko A, Greschner M, Sher A, Litke AM, Chichilnisky EJ, Feller MB: Direction selectivity in the retina is established independent of visual experience and cholinergic retinal waves. Neuron 2008, 58:499-506.

26. Chan Y-C, Chiao C-C: Effect of visual experience on the maturation of ONOFF direction selective ganglion cells in the rabbit retina. Vision Res. 2008, 48:2466-2475.

27. Chen M, Weng S, Deng Q, Xu Z, He S: Physiological properties of directionselective ganglion cells in early postnatal and adult mouse retina. The Journal of Physiology 2009, 587:819-828.

28. Sun L, Han X, He S: Direction-selective circuitry in rat retina develops independently of GABAergic, cholinergic and action potential activity. PLoS ONE 2011, 6:e19477.

29. Dhande OS, Stafford BK, Lim J-HA, Huberman AD: Contributions of Retinal Ganglion Cells to Subcortical Visual Processing and Behaviors. Annu. Rev. Vis. Sci. 2015, 1:291-328.

30. Morrie RD, Feller MB: An Asymmetric Increase in Inhibitory Synapse Number Underlies the Development of a Direction Selective Circuit in the Retina. J. Neurosci. 2015, 35:9281-9286.

31. Yonehara K, Balint K, Noda M, Nagel G, Bamberg E, Roska B: Spatially asymmetric reorganization of inhibition establishes a motion-sensitive circuit. Nature 2011, 469:407-410.

32. Elstrott J, Feller MB: Vision and the establishment of direction-selectivity: a tale of two circuits. Current Opinion in Neurobiology 2009, 19:293-297.

33. Kim I-J, Zhang Y, Meister M, Sanes JR: Laminar restriction of retinal ganglion cell dendrites and axons: subtype-specific developmental patterns revealed with transgenic markers. J. Neurosci. 2010, 30:1452-1462.

34. ••Kay JN, la Huerta De I, Kim I-J, Zhang Y, Yamagata M, Chu MW, Meister M, Sanes JR: Retinal ganglion cells with distinct directional preferences differ in molecular identity, structure, and central projections. J. Neurosci. 2011, 31:7753-7762.

This study was the first to identify genetic markers specific to different subpopulations of DSGCs using microarray analysis.

35. Huberman AD, Wei W, Elstrott J, Stafford BK, Feller MB, Barres BA: Genetic identification of an On-Off direction-selective retinal ganglion cell subtype 
reveals a layer-specific subcortical map of posterior motion. Neuron 2009, 62:327-334.

36. la Huerta De I, Kim I-J, Voinescu PE, Sanes JR: Direction-selective retinal ganglion cells arise from molecularly specified multipotential progenitors. Proceedings of the National Academy of Sciences 2012, 109:17663-17668.

37. Lefebvre JL, Kostadinov D, Chen WV, Maniatis T, Sanes JR: Protocadherins mediate dendritic self-avoidance in the mammalian nervous system. Nature 2012, 488:517-521.

38. ••Sun LO, Jiang Z, Rivlin-Etzion M, Hand R, Brady CM, Matsuoka RL, Yau KW, Feller MB, Kolodkin AL: On and Off Retinal Circuit Assembly by Divergent Molecular Mechanisms. Science 2013, 342:1241974-1241974.

This study found that ON SACs, but not OFF SACs, express the transmembrane protein Sema6A, and that this expression pattern mediates the different dendritic stratification patterns of the two SAC populations via repulsive signaling with PlexA2. Furthermore, the authors show that Sema6A is required for proper ON SAC dendritic morphology and that DSGCs have reduced ON, but not $\mathrm{OFF}$, direction selectivity in Sema6 $\mathrm{A}^{-/-}$mice.

39. Lefebvre JL, Sanes JR, Kay JN: Development of Dendritic Form and Function. Annu. Rev. Cell Dev. Biol. 2015, doi:10.1146/annurev-cellbio-100913-013020.

40. $\bullet$ Kostadinov D, Sanes JR: Protocadherin-dependent dendritic self-avoidance regulates neural connectivity and circuit function. Elife 2015, 4.

This study demonstrates an essential role for SAC self/non-self discrimination in DS using transgenic mouse lines to manipulate Pcdhg expression in SACs. In addition, the authors are the first to show a refinement in GABAergic SAC-SAC connectivity during development.

41. Koropouli E, Kolodkin AL: Semaphorins and the dynamic regulation of synapse assembly, refinement, and function. Current Opinion in Neurobiology 2014, 27C:1-7.

42. Matsuoka RL, Nguyen-Ba-Charvet KT, Parray A, Badea TC, Chédotal A, Kolodkin AL: Transmembrane semaphorin signalling controls laminar stratification in the mammalian retina. Nature 2011, 470:259-263.

43. Thomas S, Proudlock FA, Sarvananthan N, Roberts EO, Awan M, McLean R, Surendran M, Anil Kumar AS, Farooq SJ, Degg C, et al.: Phenotypical characteristics of idiopathic infantile nystagmus with and without mutations in FRMD7. Brain 2007, 131:1259-1267.

44. $•$ Yonehara K, Fiscella M, Drinnenberg A, Esposti F, Trenholm S, Krol J, Franke F, Scherf BG, Kusnyerik A, Müller J, et al.: Congenital Nystagmus Gene 
FRMD7 Is Necessary for Establishing a Neuronal Circuit Asymmetry for Direction Selectivity. Neuron 2015, doi:10.1016/j.neuron.2015.11.032.

This pioneering study found that the lack of horizontal optokinetic reflexes in human patients with FRMD7 mutations was phenocopied by mice with a hypomorphic mutation in FRMD7. The authors explained this phenotype by demonstrating a specific loss of horizontal DSGCs in Frmd $7^{\mathrm{tm}}$ mice using MEA recordings of explanted retinas. Genetically labeled horizontal DSGCs lost DS due to an increase in preferred direction inhibition, which prevents spiking to all directions of moving stimuli.

45. Siegert S, Cabuy E, Scherf BG, Kohler H, Panda S, Le Y-Z, Fehling HJ, Gaidatzis D, Stadler MB, Roska B: Transcriptional code and disease map for adult retinal cell types. Nat Neurosci 2012, 15:487-495.

46. Pu J, Mao Y, Lei X, Yan Y, Lu X, Tian J, Yin X, Zhao G, Zhang B: FERM domain containing protein 7 interacts with the Rho GDP dissociation inhibitor and specifically activates Rac1 signaling. PLOS ONE 2013, 8:e73108.

47. Huang ZJ: Activity-dependent development of inhibitory synapses and innervation pattern: role of GABA signalling and beyond. The Journal of Physiology 2009, 587:1881-1888.

48. Hamby AM, Rosa JM, Hsu C-H, Feller MB: CaV3.2 KO mice have altered retinal waves but normal direction selectivity. Vis. Neurosci. 2015, 32:E003.

49. Elstrott J, Feller MB: Direction-selective ganglion cells show symmetric participation in retinal waves during development. J. Neurosci. 2010, 30:11197-11201.

50. Stafford BK, Sher A, Litke AM, Feldheim DA: Spatial-temporal patterns of retinal waves underlying activity-dependent refinement of retinofugal projections. Neuron 2009, 64:200-212.

51. Bloodgood BL, Sharma N, Browne HA, Trepman AZ, Greenberg ME: The activity-dependent transcription factor NPAS4 regulates domain-specific inhibition. Nature 2013, 503:121-125.

52. Chan Y-C, Chiao C-C: The distribution of the preferred directions of the ONOFF direction selective ganglion cells in the rabbit retina requires refinement after eye opening. Physiol Rep 2013, 1:e00013.

53. Chen H, Liu X, Tian N: Subtype-Dependent Postnatal Development of Direction- and Orientation-Selective Retinal Ganglion Cells in Mice. $J$. Neurophysiol. 2014, 112:2092-2101.

54. ••Bos R, Gainer C, Feller MB: Role for Visual Experience in the Development of Direction-Selective Circuits. Current Biology 2016, 
doi:10.1016/j.cub.2016.03.073.

Using 2-photon calcium imaging, the authors find that at eye opening and in dark reared adults DSGC preferred directions are diffusely distributed, demonstrating a key role for visual signaling in the clustering of DSGC preferred directions to the cardinal axes.

55. Bleckert A, Parker ED, Kang Y, Pancaroglu R, Soto F, Lewis R, Craig AM, Wong ROL: Spatial Relationships between GABAergic and Glutamatergic Synapses on the Dendrites of Distinct Types of Mouse Retinal Ganglion Cells across Development. PLoS ONE 2013, 8:e69612.

56. McLaughlin T, O'Leary DDM: Molecular gradients and development of retinotopic maps. Annu. Rev. Neurosci. 2005, 28:327-355.

57. Clandinin TR, Feldheim DA: Making a visual map: mechanisms and molecules. Current Opinion in Neurobiology 2009, 19:174-180.

58. Visser JJ, Cheng Y, Perry SC, Chastain AB, Parsa B, Masri SS, Ray TA, Kay JN, Wojtowicz WM: An extracellular biochemical screen reveals that FLRTs and Unc5s mediate neuronal subtype recognition in the retina. Elife 2015, 4:e08149.

59. Krishnaswamy A, Yamagata M, Duan X, Hong YK, Sanes JR: Sidekick 2 directs formation of a retinal circuit that detects differential motion. Nature 2015, 524:466-470.

60. Tang JCY, Szikra T, Kozorovitskiy Y, Teixiera M, Sabatini BL, Roska B, Cepko CL: A nanobody-based system using fluorescent proteins as scaffolds for cellspecific gene manipulation. Cell 2013, 154:928-939.

61. Kim I-J, Zhang Y, Yamagata M, Meister M, Sanes JR: Molecular identification of a retinal cell type that responds to upward motion. Nature 2008, 452:478482.

62. Baden T, Berens P, Franke K, Rosón MR, Bethge M, Euler T: The functional diversity of retinal ganglion cells in the mouse. Nature 2016, 529:345-350.

63. Joesch M, Meister M: A neuronal circuit for colour vision based on rod-cone opponency. Nature 2016, 532:236-239. 
Figure 1
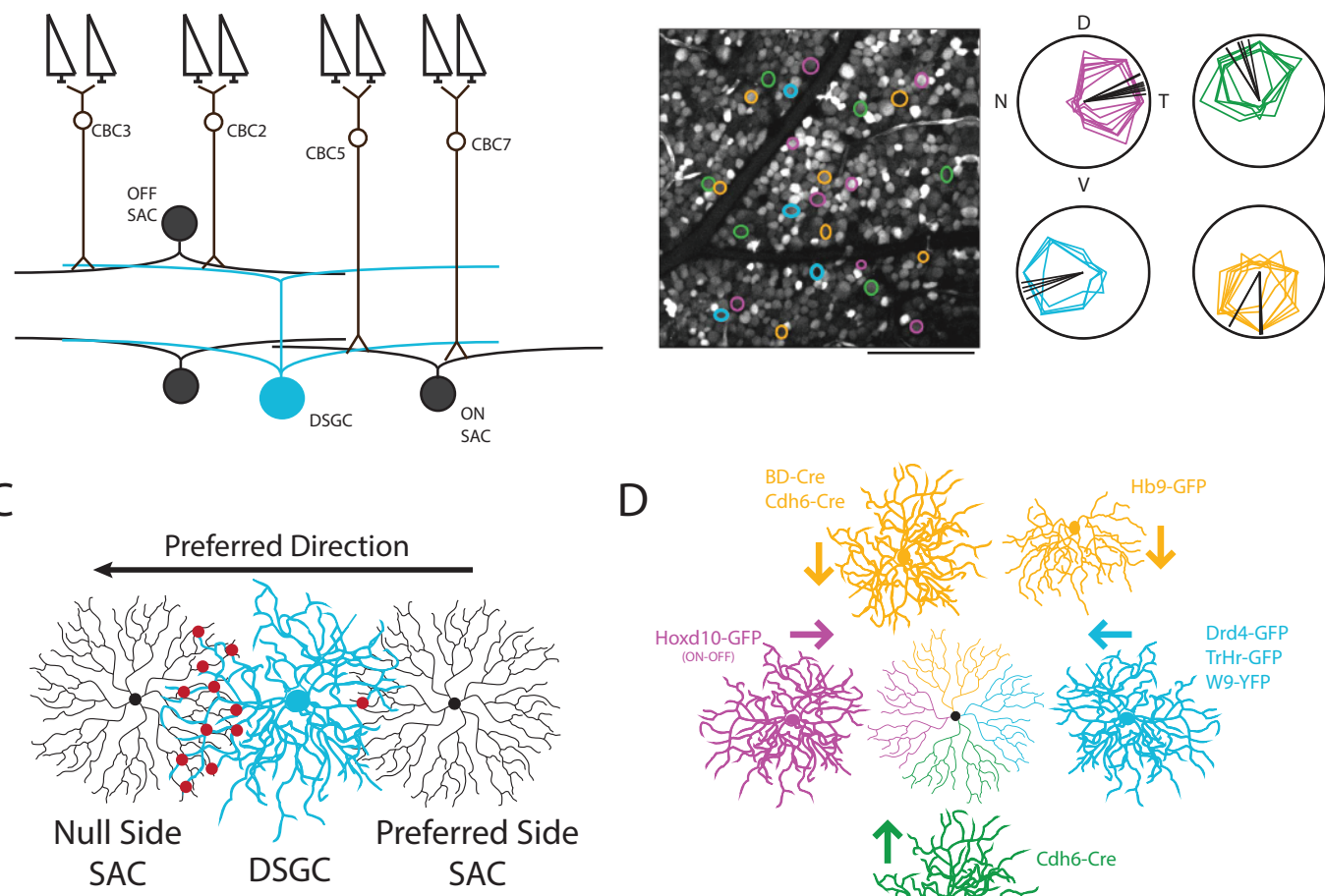

D

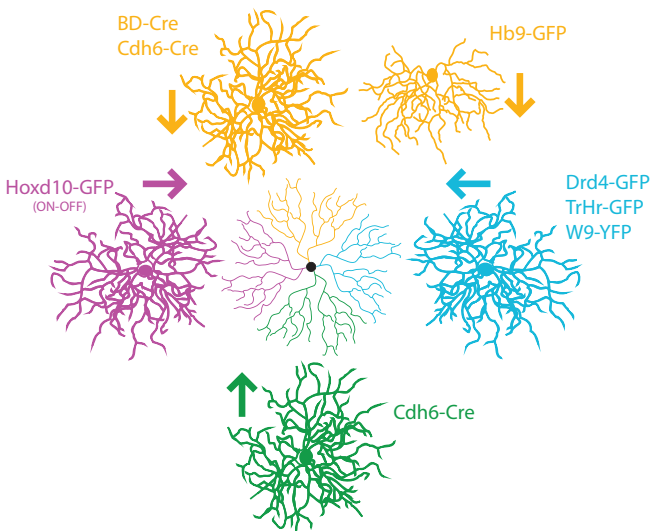


SAC Morphology and Connectivity

Figure 2

A
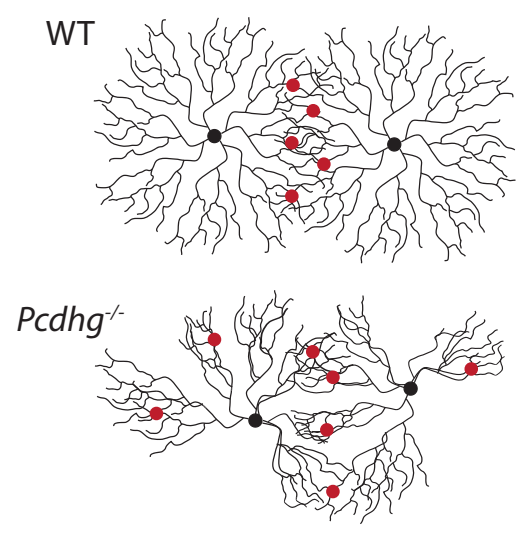

$\mathrm{C}$
$\mathrm{D}$

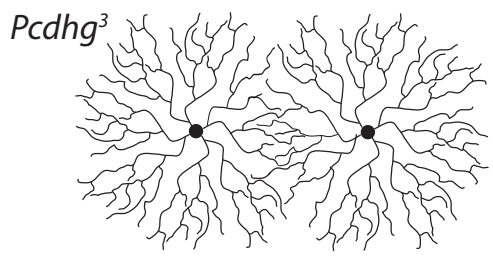

Sema6 $A^{-1-}$

ON SACS

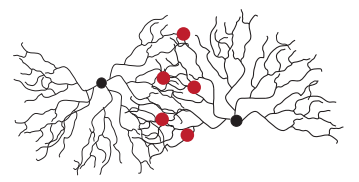

$E$

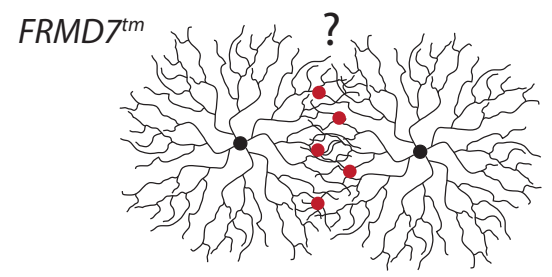
ON response

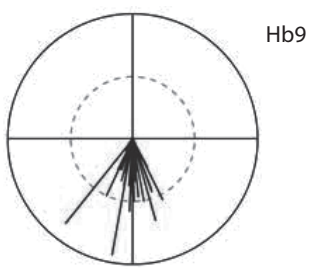

OFF response
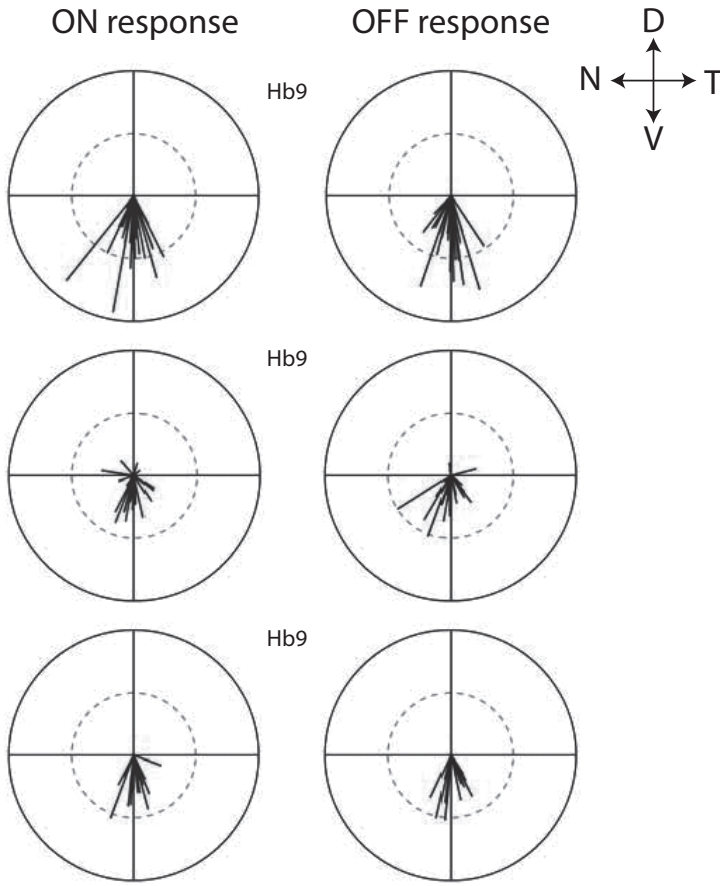

$\mathrm{Hb} 9$

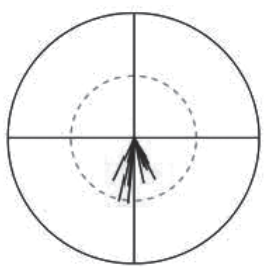

$\mathrm{TrHr}$
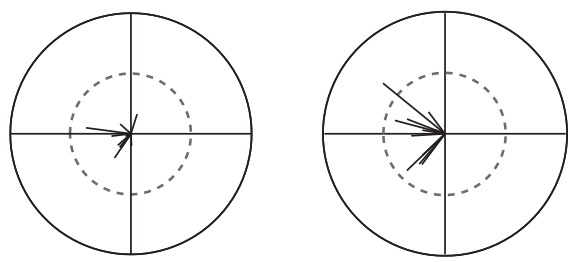

whole retina MEA

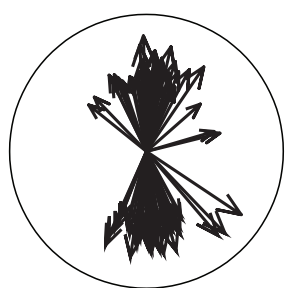

\section{cell-attached}

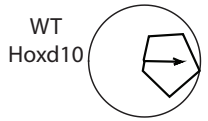

FRMD7 ${ }^{\text {tm }}$

Hoxd10 
Eye Opening / Dark Reared Adult

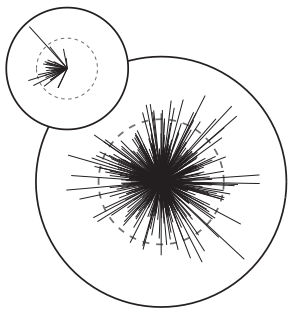

Eye Opening
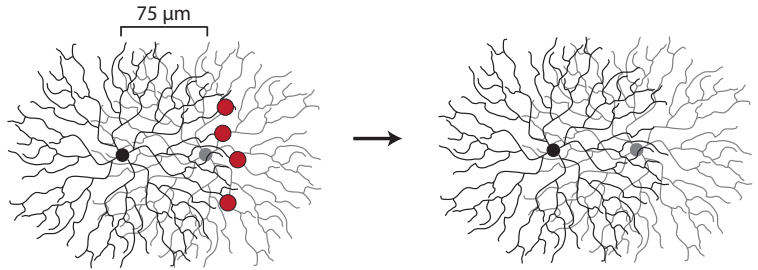

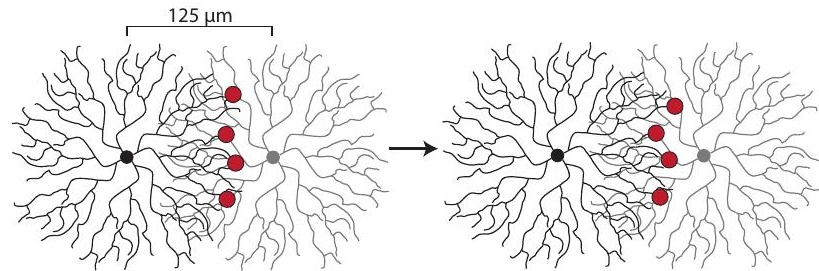

